\title{
SEQUENCES OF WEAK SOLUTIONS FOR FRACTIONAL EQUATIONS
}

\author{
Giovanni Molica Bisci
}

\begin{abstract}
This work is devoted to study the existence of infinitely many weak solutions to nonocal equations involving a general integrodifferential operator of fractional type. These equations have a variational structure and we find a sequence of nontrivial weak solutions for them exploiting the $\mathbb{Z}_{2}$-symmetric version of the Mountain Pass Theorem. To make the nonlinear methods work, some careful analysis of the fractional spaces involved is necessary. As a particular case, we derive an existence theorem for the fractional Laplacian, finding nontrivial solutions of the equation
\end{abstract}

$$
\begin{cases}(-\Delta)^{s} u=f(x, u) & \text { in } \Omega, \\ u=0 & \text { in } \mathbb{R}^{n} \backslash \Omega .\end{cases}
$$

As far as we know, all these results are new and represent a fractional version of classical theorems obtained working with Laplacian equations.

\section{Introduction}

In this paper, we obtain an existence result for the following nonlocal problem:

$$
\begin{cases}-\mathcal{L}_{K} u=f(x, u) & \text { in } \Omega, \\ u=0 & \text { in } \mathbb{R}^{n} \backslash \Omega .\end{cases}
$$

Here and in the sequel, $\Omega$ is a bounded domain in $\left(\mathbb{R}^{n},|\cdot|\right)$ with $n>2 s$ (where $s \in(0,1)$ ) and with smooth (Lipschitz) boundary $\partial \Omega, f: \bar{\Omega} \times \mathbb{R} \rightarrow \mathbb{R}$ is a suitable continuous function with subcritical growth and $\mathcal{L}_{K}$ is the nonlocal operator defined as follows:

$$
\mathcal{L}_{K} u(x):=\int_{\mathbb{R}^{n}}(u(x+y)+u(x-y)-2 u(x)) K(y) d y, \quad\left(x \in \mathbb{R}^{n}\right),
$$

where $K: \mathbb{R}^{n} \backslash\{0\} \rightarrow(0,+\infty)$ is a function with the properties that:

$\left(k_{1}\right) \gamma K \in L^{1}\left(\mathbb{R}^{n}\right)$, where $\gamma(x)=\min \left\{|x|^{2}, 1\right\}$;

$\left(k_{2}\right)$ There exists $\lambda>0$ such that

$$
K(x) \geq \lambda|x|^{-(n+2 s)},
$$

for any $x \in \mathbb{R}^{n} \backslash\{0\}$;

$\left(k_{3}\right) K(x)=K(-x)$, for any $x \in \mathbb{R}^{n} \backslash\{0\}$.

Received by the editors July 25, 2013.

2010 Mathematics Subject Classification. Primary: 49J35, 35S15; Secondary: 47G20, 45 G05.

Key words and phrases. Nonlocal problems; Fractional Equations; Mountain Pass Theorem. 
A typical example for the kernel $K$ is given by $K(x):=|x|^{-(n+2 s)}$. In this case $\mathcal{L}_{K}$ is the fractional Laplace operator defined as

$$
-(-\Delta)^{s} u(x):=\int_{\mathbb{R}^{n}} \frac{u(x+y)+u(x-y)-2 u(x)}{|y|^{n+2 s}} d y, \quad x \in \mathbb{R}^{n} .
$$

Recently, a great attention has been focused on the study of fractional and nonlocal operators of elliptic type, both for the pure mathematical research and in view of concrete real-world applications. This type of operators arises in a quite natural way in many different contexts, such as, among the others, the thin obstacle problem, optimization, finance, phase transitions, stratified materials, anomalous diffusion, crystal dislocation, soft thin films, semipermeable membranes, flame propagation, conservation laws, ultra-relativistic limits of quantum mechanics, quasi-geostrophic flows, multiple scattering, minimal surfaces, materials science and water waves.

In this paper, exploiting classical variational methods, problem $\left(P_{K}^{f}\right)$ is studied requiring that the associated energy functional satisfies a standard symmetry assumption.

More precisely, we apply the $\mathbb{Z}_{2}$-symmetric version of the Mountain Pass Theorem (briefly $\mathbb{Z}_{2}$-MPT) for even functionals (see [11, Theorem 9.12]) to this kind of equations motivated by the current literature where the MPT has been intensively applied to find solutions to quasilinear elliptic equations; see [2, 10, 11, 18].

Technically, this approach is realizable checking that the associated energy functional verifies the usual compactness Palais-Smale (PS) condition in a suitable variational setting developed in [14]. Indeed, the nonlocal analysis that we perform here in order to use Mountain Pass Theorem is quite general and successfully exploited for other goals in several recent contributions; see $[4,14,15,17]$ for an elementary introduction to this topic and for a list of related references.

This functional analytical context is inspired by (but not equivalent to) the fractional Sobolev spaces, in order to correctly encode the Dirichlet boundary datum in the variational formulation.

Further, we suppose that the right-hand side of equation $\left(P_{K}^{f}\right)$ is a continuous odd function $f: \bar{\Omega} \times \mathbb{R}^{n} \rightarrow \mathbb{R}$ verifying the following conditions:

$\left(h_{1}\right)$ There exist $a_{1}, a_{2}>0$ and $q \in\left(2,2^{*}\right), 2^{*}:=\frac{2 n}{n-2 s}$ such that

$$
|f(x, t)| \leq a_{1}+a_{2}|t|^{q-1}
$$

for every $x \in \bar{\Omega}, t \in \mathbb{R}$;

$\left(h_{2}\right)$ There are two constants $\theta>2$ and $r>0$ such that

$$
0<\theta F(x, t) \leq t f(x, t),
$$

for every $x \in \bar{\Omega}$, and $|t| \geq r$,

where the function $F$ is the primitive of $f$ with respect to the second variable, that is

$$
F(x, t):=\int_{0}^{t} f(x, s) d s, \quad(\forall t \in \mathbb{R}) .
$$

Under the previous assumptions, we prove the existence of infinitely many weak solutions to problem $\left(P_{K}^{f}\right)$; see Theorem 3.1. Note that the symmetry hypothesis on $f$ allows to remove any condition at zero. 
In the nonlocal framework, the simplest example we can deal with is given by the fractional Laplacian, according to the following proposition.

Theorem 1.1. Let $s \in(0,1), n>2 s$ and $\Omega$ be an open bounded set of $\mathbb{R}^{n}$ with Lipschitz boundary.

Consider the following equation:

$$
\int_{\mathbb{R}^{n} \times \mathbb{R}^{n}} \frac{(u(x)-u(y))(\varphi(x)-\varphi(y))}{|x-y|^{n+2 s}} d x d y=\int_{\Omega} f(x, u(x)) \varphi(x) d x
$$

for any $\varphi \in H^{s}\left(\mathbb{R}^{n}\right)$ with $\varphi=0$ a.e. in $\mathbb{R}^{n} \backslash \Omega$.

If $f: \bar{\Omega} \times \mathbb{R} \rightarrow \mathbb{R}$ is an odd continuous function verifying $\left(h_{1}\right)$ and $\left(h_{2}\right)$, then problem (1.2) admits a sequence of infinitely many solutions $\left\{u_{j}\right\}_{j \in \mathbb{N}} \subset H^{s}\left(\mathbb{R}^{n}\right)$, such that $u_{j}=0$ a.e. in $\mathbb{R}^{n} \backslash \Omega$.

The above result is the fractional analogous of [11, Theorem 9.38] in which the classical Dirichlet problem

$$
\begin{cases}-\Delta u=f(x, u) & \text { in } \Omega, \\ u=0 & \text { on } \partial \Omega,\end{cases}
$$

is studied; see [18, Theorem 6.6]. We also cite $[2,10]$ where MPT (and some of its variant) has been intensively applied to find solutions to quasilinear elliptic equations.

The plan of the paper is as follows; Section 2 is devoted to our abstract framework and preliminaries. Successively, in Section 3 we give the main result; see Theorem 3.1. Finally, a concrete example of application is presented in Example 3.6.

\section{Abstract framework}

In this subsection, we briefly recall the definition of the functional space $X_{0}$, firstly introduced in [13], and we give some notations. The reader familiar with this topic may skip this section and go directly to the next one.

The functional space $X$ denotes the linear space of Lebesgue measurable functions from $\mathbb{R}^{n}$ to $\mathbb{R}$ such that the restriction to $\Omega$ of any function $g$ in $X$ belongs to $L^{2}(\Omega)$ and

$$
((x, y) \mapsto(g(x)-g(y)) \sqrt{K(x-y)}) \in L^{2}\left(\left(\mathbb{R}^{n} \times \mathbb{R}^{n}\right) \backslash(\mathcal{C} \Omega \times \mathcal{C} \Omega), d x d y\right),
$$

where $\mathcal{C} \Omega:=\mathbb{R}^{n} \backslash \Omega$.

We denote by $X_{0}$ the following linear subspace of $X$

$$
X_{0}:=\left\{g \in X: g=0 \text { a.e. in } \mathbb{R}^{n} \backslash \Omega\right\} .
$$

We remark that $X$ and $X_{0}$ are nonempty, since $C_{0}^{2}(\Omega) \subseteq X_{0}$ by [13, Lemma 11]. Moreover, the space $X$ is endowed with the norm defined as

$$
\|g\|_{X}:=\|g\|_{L^{2}(\Omega)}+\left(\int_{Q}|g(x)-g(y)|^{2} K(x-y) d x d y\right)^{1 / 2},
$$

where $Q:=\left(\mathbb{R}^{n} \times \mathbb{R}^{n}\right) \backslash \mathcal{O}$ and $\mathcal{O}:=(\mathcal{C} \Omega) \times(\mathcal{C} \Omega) \subset \mathbb{R}^{n} \times \mathbb{R}^{n}$.

It is easily seen that $\|\cdot\|_{X}$ is a norm on $X$; see, for instance, [14] for a proof. 
By $[14$, Lemmas 6 and 7] in the sequel we can take the function

$$
X_{0} \ni v \mapsto\|v\|_{X_{0}}:=\left(\int_{Q}|v(x)-v(y)|^{2} K(x-y) d x d y\right)^{1 / 2}
$$

as norm on $X_{0}$.

Also $\left(X_{0},\|\cdot\|_{X_{0}}\right)$ is a Hilbert space with scalar product

$$
\langle u, v\rangle_{X_{0}}:=\int_{Q}(u(x)-u(y))(v(x)-v(y)) K(x-y) d x d y,
$$

see [14, Lemma 7].

Note that in (2.1) (and in the related scalar product) the integral can be extended to all $\mathbb{R}^{n} \times \mathbb{R}^{n}$, since $v \in X_{0}$ (and so $v=0$ a.e. in $\mathbb{R}^{n} \backslash \Omega$ ).

In what follows, we denote by $\lambda_{k}$ be the $k$-th eigenvalue of the operator $-\mathcal{L}_{K}$ with homogeneous Dirichlet boundary data, namely the $k$-th eigenvalue of the problem

$$
\begin{cases}-\mathcal{L}_{K} u=\lambda u & \text { in } \Omega \\ u=0 & \text { in } \mathbb{R}^{n} \backslash \Omega .\end{cases}
$$

The set of the eigenvalues of problem (2.2) consists of a sequence $\left\{\lambda_{k}\right\}_{k \in \mathbb{N}}$ with $^{1}$

$$
0<\lambda_{1}<\lambda_{2} \leq \cdots \leq \lambda_{k} \leq \lambda_{k+1} \leq \cdots
$$

and

$$
\lambda_{k} \rightarrow+\infty \text { as } k \rightarrow \infty
$$

Further, the following characterization holds:

$$
\lambda_{k}=\min _{u \in \mathbb{P}_{k} \backslash\left\{0_{X_{0}}\right\}} \frac{\int_{\mathbb{R}^{2 n}}|u(x)-u(y)|^{2} K(x-y) d x d y}{\int_{\Omega} u(x)^{2} d x},
$$

where

$$
\mathbb{P}_{k}:=\left\{u \in X_{0}:\left\langle u, e_{j}\right\rangle_{X_{0}}=0 \quad \forall j=1, \ldots, k\right\} .
$$

Finally, the symbol $E\left(\lambda_{k}\right)$ denotes the linear space of eigenfunctions corresponding to $\lambda_{k}$. For the existence and the basic properties of these eigenvalues we refer to [15, Proposition 9 and Appendix A], where a spectral theory for these general integrodifferential nonlocal operators was developed. Further properties can be also found in [12].

While for a general kernel $K$ satisfying conditions from $\left(k_{1}\right)$ to $\left(k_{3}\right)$ we have that $X_{0} \subset H^{s}\left(\mathbb{R}^{n}\right)$, in the model case $K(x):=|x|^{-(n+2 s)}$ the space $X_{0}$ consists of all the functions of the usual fractional Sobolev space $H^{s}\left(\mathbb{R}^{n}\right)$ which vanish a.e. outside $\Omega$; see $[17$, Lemma 7].

Here $H^{s}\left(\mathbb{R}^{n}\right)$ denotes the usual fractional Sobolev space endowed with the norm (the so-called Gagliardo norm)

$$
\|g\|_{H^{s}\left(\mathbb{R}^{n}\right)}=\|g\|_{L^{2}\left(\mathbb{R}^{n}\right)}+\left(\int_{\mathbb{R}^{n} \times \mathbb{R}^{n}} \frac{|g(x)-g(y)|^{2}}{|x-y|^{n+2 s}} d x d y\right)^{1 / 2} .
$$

\footnotetext{
${ }^{1}$ As usual, here we call $\lambda_{1}$ the first eigenvalue of the operator $-\mathcal{L}_{K}$. This notation is justified by (2.3). Notice also that some of the eigenvalues in the sequence $\left\{\lambda_{k}\right\}_{k \in \mathbb{N}}$ may repeat, i.e., the inequalities in (2.3) may be not always strict.
} 
Before concluding this subsection, we recall the embedding properties of $X_{0}$ into the usual Lebesgue spaces; see [14, Lemma 8].

The embedding $j: X_{0} \hookrightarrow L^{\nu}\left(\mathbb{R}^{n}\right)$ is continuous for any $\nu \in\left[1,2^{*}\right]$, while it is compact whenever $\nu \in\left[1,2^{*}\right)$, where $2^{*}:=2 n /(n-2 s)$. Hence, for any $\nu \in\left[1,2^{*}\right]$ there exists a positive constant $c_{\nu}$ such that

$$
\|v\|_{L^{\nu}\left(\mathbb{R}^{n}\right)} \leq c_{\nu}\|v\|_{X_{0}} \text { for any } v \in X_{0} .
$$

For further details on the fractional Sobolev spaces we refer to [4] and to the references therein, while for other details on $X$ and $X_{0}$ we refer to [13], where these functional spaces were introduced, and also to $[12,14,15,17]$, where various properties of these spaces were proved.

Finally, for the sake of completeness, we recall that a $C^{1}$-functional $J: E \rightarrow \mathbb{R}$, where $E$ is a real Banach space with topological dual $E^{*}$, satisfies the Palais-Smale condition at level $\mu \in \mathbb{R}$, (briefly $(\mathrm{PS})_{\mu}$ ) when:

$(\mathrm{PS})_{\mu}$ Every sequence $\left\{u_{j}\right\}_{j \in \mathbb{N}}$ in $E$ such that

$$
J\left(u_{j}\right) \rightarrow \mu, \text { and }\left\|J^{\prime}\left(u_{j}\right)\right\|_{E^{*}} \rightarrow 0,
$$

as $j \rightarrow \infty$, possesses a convergent subsequence.

We say that $J$ satisfies the Palais-Smale condition (in short (PS)) if (PS) ${ }_{\mu}$ holds for every $\mu \in \mathbb{R}$.

With the above notation, our main tool is the following classical result recalled in a convenient form.

Theorem 2.1. Let $E$ be an infinite-dimensional real Banach space and let $J \in$ $C^{1}(E ; \mathbb{R})$ be even, satisfying the (PS) condition and $J\left(0_{E}\right)=0$. Suppose $E=E_{1} \oplus E_{2}$, where $E_{1}$ is finite-dimensional and $J$ satisfies:

$\left(I_{1}\right)$ There exist constant $\rho, \alpha>0$ such that

$$
J(u) \geq \alpha,
$$

for every $u \in E_{2}$ and $\|u\|_{E}=\rho$.

$\left(I_{2}\right)$ For each finite-dimensional subspace $W \subset E$, the set

$$
\{u \in W: J(u) \geq 0\}
$$

is bounded in E.

Then $J$ has an unbounded sequence of critical values.

See [11, Theorem 9.12].

We cite the monograph [6] as general reference on variational methods adopted in this paper.

\section{The main theorem}

Our result is as follows.

Theorem 3.1. Let $s \in(0,1), n>2 s$ and $\Omega$ be an open bounded set of $\mathbb{R}^{n}$ with Lipschitz boundary and $K: \mathbb{R}^{n} \backslash\{0\} \rightarrow(0,+\infty)$ be a map satisfying $\left(k_{1}\right)-\left(k_{3}\right)$. In addition, let $f: \bar{\Omega} \times \mathbb{R} \rightarrow \mathbb{R}$ be an odd continuous function verifying $\left(h_{1}\right)$ and $\left(h_{2}\right)$. Then, problem $\left(P_{K}^{f}\right)$ possesses an unbounded sequence of weak solutions. 
We recall that a weak solution of problem $\left(P_{K}^{f}\right)$ is a function $u \in X_{0}$ such that

$$
\begin{gathered}
\int_{Q}(u(x)-u(y))(\varphi(x)-\varphi(y)) K(x-y) d x d y \\
=\int_{\Omega} f(x, u(x)) \varphi(x) d x, \quad \forall \varphi \in X_{0} .
\end{gathered}
$$

For the proof of Theorem 3.1, we observe that problem $\left(P_{K}^{f}\right)$ has a variational structure, indeed it is the Euler-Lagrange equation of the functional $J_{K}: X_{0} \rightarrow \mathbb{R}$ defined as follows:

$$
J_{K}(u):=\frac{1}{2} \int_{Q}|u(x)-u(y)|^{2} K(x-y) d x d y-\int_{\Omega} F(x, u(x)) d x .
$$

Note that the functional $J_{K}$ is Fréchet differentiable in $u \in X_{0}$ and for any $\varphi \in X_{0}$ one has

$$
\left\langle J_{K}^{\prime}(u), \varphi\right\rangle=\int_{Q}(u(x)-u(y))(\varphi(x)-\varphi(y)) K(x-y) d x d y-\int_{\Omega} f(x, u(x)) \varphi(x) d x .
$$

Thus, critical points of $J_{K}$ are solutions to problem $\left(P_{K}^{f}\right)$. In order to find these critical points, we will make use of Theorem 2.1. For this, we have to check that the functional $J_{K}$ has a particular geometric structure and satisfies the PS compactness condition.

3.1. Proof of Theorem 3.1. In order to prove our result, we apply (as claimed before) Theorem 2.1 to the functional $J_{K}$ defined in (3.1). The conclusion of Theorem 3.1 is equivalent to the assertion that $J_{K}$ admits an unbounded sequence of critical points. Hence, let us start observing that, since $f$ is odd in the second variable i.e., $f(x,-t)=-f(x, t)$, for every $t \in \mathbb{R}, J_{K}$ is even. Moreover, by definition, $J_{K}\left(0_{X_{0}}\right)=0$.

In the next two lemmas, we shall verify the compactness (PS) condition.

Lemma 3.2. Every PS sequence for the functional $J_{K}$ is bounded in $X_{0}$.

Proof. Let $\left\{u_{j}\right\}_{j \in \mathbb{N}} \subset X_{0}$ be a PS sequence i.e.,

$$
J_{K}\left(u_{j}\right) \rightarrow \mu,
$$

for $\mu \in \mathbb{R}$ and

$$
\left\|J_{K}^{\prime}\left(u_{j}\right)\right\|_{X_{0}^{*}}=\sup \left\{\left|\left\langle J_{K}^{\prime}\left(u_{j}\right), \varphi\right\rangle\right|: \varphi \in X_{0},\|\varphi\|_{X_{0}}=1\right\} \rightarrow 0,
$$

as $j \rightarrow \infty$.

We argue by contradiction. So, suppose that the conclusion is not true. Passing to a subsequence if necessary, we may assume that

$$
\left\|u_{j}\right\|_{X_{0}} \rightarrow+\infty
$$

as $j \rightarrow \infty$. 
By definition it follows that

$$
\begin{aligned}
J_{K}\left(u_{j}\right)-\frac{\left\langle J_{K}^{\prime}\left(u_{j}\right), u_{j}\right\rangle}{\theta}= & \left(\frac{1}{2}-\frac{1}{\theta}\right) \int_{Q}\left|u_{j}(x)-u_{j}(y)\right|^{2} K(x-y) d x d y \\
& +\int_{\Omega}\left[\frac{f\left(x, u_{j}(x)\right) u_{j}(x)}{\theta}-F\left(x, u_{j}(x)\right)\right] d x
\end{aligned}
$$

for every $j \in \mathbb{N}$.

Thus

$$
\begin{aligned}
\left(\frac{\theta-2}{2 \theta}\right)\left\|u_{j}\right\|_{X_{0}}^{2} \leq & J_{K}\left(u_{j}\right)-\frac{\left\langle J_{K}^{\prime}\left(u_{j}\right), u_{j}\right\rangle}{\theta} \\
& -\int_{\left|u_{j}(x)\right|>r}\left[\frac{f\left(x, u_{j}(x)\right) u_{j}(x)}{\theta}-F\left(x, u_{j}(x)\right)\right] d x, \\
& +M \operatorname{meas}(\Omega), \quad \forall j \in \mathbb{N},
\end{aligned}
$$

where "meas $(\Omega)$ " denotes the standard Lebesgue measure of $\Omega$ and

$$
M:=\max \left\{\left|\frac{f(x, t) t}{\theta}-F(x, t)\right|: x \in \bar{\Omega},|t| \leq r\right\} .
$$

Now, we observe that, the Ambrosetti Rabinowitz condition yields

$$
\int_{\left|u_{j}(x)\right|>r}\left[\frac{f\left(x, u_{j}(x)\right) u_{j}(x)}{\theta}-F\left(x, u_{j}(x)\right)\right] d x \geq 0 .
$$

So, we deduce that

$$
\left(\frac{\theta-2}{2 \theta}\right)\left\|u_{j}\right\|_{X_{0}}^{2} \leq J_{K}\left(u_{j}\right)-\frac{\left\langle J_{K}^{\prime}\left(u_{j}\right), u_{j}\right\rangle}{\theta}+M \operatorname{meas}(\Omega),
$$

for every $j \in \mathbb{N}$.

Then, for every $j \in \mathbb{N}$ one has

$$
C\left\|u_{j}\right\|_{X_{0}}^{2} \leq J_{K}\left(u_{j}\right)+\theta\left\|J_{K}^{\prime}\left(u_{j}\right)\right\|_{X_{0}^{*}}\left\|u_{j}\right\|_{X_{0}}+M \operatorname{meas}(\Omega),
$$

where $C:=m_{0}\left(\frac{\theta-2}{2 \theta}\right)>0$.

In conclusion, dividing by $\left\|u_{j}\right\|_{X_{0}}$ and letting $j \rightarrow \infty$, we obtain a contradiction.

The above Lemma implies that the $C^{1}$-functional $J_{K}$ verifies the PS condition as proved in the next result.

Lemma 3.3. The functional $J_{K}$ satisfies the compactness (PS) condition.

Proof. Let $\left\{u_{j}\right\}_{j \in \mathbb{N}} \subset X_{0}$ be a PS sequence and, in order to simplifies the notations, let us put

$$
\Phi(u):=\frac{1}{2} \int_{Q}|u(x)-u(y)|^{2} K(x-y) d x d y,
$$

for every $u \in X_{0}$. 
Taking into account Lemma 3.2, the sequence $\left\{u_{j}\right\}_{j \in \mathbb{N}}$ is necessarily bounded in $X_{0}$. Since $X_{0}$ is reflexive, we may extract a subsequence that for simplicity we call again $\left\{u_{j}\right\}_{j \in \mathbb{N}}$, such that $u_{j} \rightarrow u_{\infty}$ in $X_{0}$. This means that

$$
\begin{aligned}
\int_{Q} & \left(u_{j}(x)-u_{j}(y)\right)(\varphi(x)-\varphi(y)) K(x-y) d x d y \\
& \rightarrow \int_{Q}\left(u_{\infty}(x)-u_{\infty}(y)\right)(\varphi(x)-\varphi(y)) K(x-y) d x d y
\end{aligned}
$$

for any $\varphi \in X_{0}$, as $j \rightarrow \infty$.

We will prove that $u_{j}$ strongly converges to $u_{\infty} \in X_{0}$. Exploiting the derivative $J_{K}^{\prime}\left(u_{j}\right)\left(u_{j}-u_{\infty}\right)$, we obtain

$$
\left\langle\Phi^{\prime}\left(u_{j}\right), u_{j}-u_{\infty}\right\rangle=\left\langle J_{K}^{\prime}\left(u_{j}\right), u_{j}-u_{\infty}\right\rangle+\int_{\Omega} f\left(x, u_{j}(x)\right)\left(u_{j}-u_{\infty}\right)(x) d x,
$$

where

$$
\begin{aligned}
\left\langle\Phi^{\prime}\left(u_{j}\right), u_{j}-u_{\infty}\right\rangle= & \int_{Q}\left|u_{j}(x)-u_{j}(y)\right|^{2} K(x-y) d x d y \\
& -\int_{Q}\left(u_{j}(x)-u_{j}(y)\right)\left(u_{\infty}(x)-u_{\infty}(y)\right) K(x-y) d x d y .
\end{aligned}
$$

Since $\left\|J_{K}^{\prime}\left(u_{j}\right)\right\|_{X_{0}^{*}} \rightarrow 0$ and the sequence $\left\{u_{j}-u_{\infty}\right\}$ is bounded in $X_{0}$, taking into account that $\left|\left\langle J_{K}^{\prime}\left(u_{j}\right), u_{j}-u_{\infty}\right\rangle\right| \leq\left\|J_{K}^{\prime}\left(u_{j}\right)\right\|_{X_{0}^{*}}\left\|u_{j}-u_{\infty}\right\|_{X_{0}}$, one has

$$
\left\langle J_{K}^{\prime}\left(u_{j}\right), u_{j}-u_{\infty}\right\rangle \rightarrow 0 \text {, }
$$

as $j \rightarrow \infty$.

At this point, we observe that, since the embedding $X_{0} \hookrightarrow L^{q}(\Omega)$ is compact, clearly $u_{j} \rightarrow u_{\infty}$ strongly in $L^{q}(\Omega)$. So by condition $\left(h_{1}\right)$, we easily obtain that

$$
\int_{\Omega}\left|f\left(x, u_{j}(x)\right)\right|\left|u_{j}(x)-u_{\infty}(x)\right| d x \rightarrow 0,
$$

as $j \rightarrow \infty$.

By (3.5) relations (3.6) and (3.7) yield

$$
\left\langle\Phi^{\prime}\left(u_{j}\right), u_{j}-u_{\infty}\right\rangle \rightarrow 0,
$$

as $j \rightarrow \infty$.

Hence by (3.8) we can write

$$
\begin{aligned}
& \int_{Q}\left|u_{j}(x)-u_{j}(y)\right|^{2} K(x-y) d x d y \\
& \quad-\int_{Q}\left(u_{j}(x)-u_{j}(y)\right)\left(u_{\infty}(x)-u_{\infty}(y)\right) K(x-y) d x d y \rightarrow 0,
\end{aligned}
$$

as $j \rightarrow \infty$.

Thus, by (3.9) and (3.4) it follows that

$$
\begin{aligned}
& \lim _{j \rightarrow \infty} \int_{Q}\left|u_{j}(x)-u_{j}(y)\right|^{2} K(x-y) d x d y \\
& =\int_{Q}\left|u_{\infty}(x)-u_{\infty}(y)\right|^{2} K(x-y) d x d y .
\end{aligned}
$$


In conclusion, thanks to [3, Proposition III.30], $u_{j} \rightarrow u_{\infty}$ in $X_{0}$. The proof is complete.

The proof of the main result is concluded if we prove that hypotheses $\left(I_{1}\right)$ and $\left(I_{2}\right)$ in Theorem 2.1 are verified. We show these facts in the next two propositions.

Proposition 3.4. The functional $J_{K}$ satisfies condition $\left(I_{1}\right)$.

Proof. We claim that there exists $k_{0} \in \mathbb{N}$ sufficiently large and two positive constants $\rho$ and $\alpha$ such that for every

$$
u \in E_{2}:=\bigoplus_{k \geq k_{0}} E\left(\lambda_{k}\right) \|^{\| \cdot X_{0}}
$$

with $\|u\|_{X_{0}}=\rho$, there holds $J_{K}(u) \geq \alpha$.

Indeed, since $q \in\left(2,2^{*}\right)$ one has

$$
\|u\|_{L^{q}(\Omega)}^{q} \leq\|u\|_{L^{2}(\Omega)}^{\beta}\|u\|_{L^{2^{*}}(\Omega)}^{q-\beta}, \quad\left(\forall u \in X_{0}\right),
$$

where we set $\beta:=2\left(\frac{2^{*}-q}{2^{*}-2}\right)$; see, for instance, $[1$, p. 105].

Now, let us denote by $\left\{e_{k}\right\}_{k \in \mathbb{N}}$ the sequence of eigenfunctions. By [15, Proposition 9 and Appendix A], we have that the sequence $\left\{e_{k}\right\}_{k \in \mathbb{N}}$ is an orthonormal basis of $L^{2}(\Omega)$ and an orthogonal basis of $X_{0}$.

More precisely, for every $k \in \mathbb{N}$ one has

$$
\left\langle e_{k}, e_{k}\right\rangle_{X_{0}}=\lambda_{k} \int_{\Omega} e_{k}(x)^{2} d x=\lambda_{k}
$$

and

$$
\left\langle e_{i}, e_{j}\right\rangle_{X_{0}}=\int_{Q}\left(e_{i}(x)-e_{i}(y)\right)\left(e_{j}(x)-e_{j}(y)\right) K(x-y) d x d y=\delta_{i}^{j}
$$

for all $i, j \in \mathbb{N}$.

If $u \in E_{2}, u=\sum_{j=k_{0}}^{+\infty} \beta_{j} e_{j}$, for suitable $\beta_{j} \in \mathbb{R}$, where $j \in \mathbb{N}$ and $j \geq k_{0}$. Hence, by using (3.11) and (3.12) one has

$$
\|u\|_{L^{2}(\Omega)}^{2}=\sum_{j=k_{0}}^{+\infty} \beta_{j}^{2} \int_{\Omega} e_{j}(x)^{2} d x=\sum_{j=k_{0}}^{+\infty} \frac{\beta_{j}^{2}}{\lambda_{j}}\left\langle e_{j}, e_{j}\right\rangle_{X_{0}} \leq \frac{1}{\lambda_{k_{0}}}\|u\|_{X_{0}}^{2},
$$

i.e.,

$$
\|u\|_{L^{2}(\Omega)} \leq \frac{1}{\sqrt{\lambda_{k_{0}}}}\|u\|_{X_{0}}
$$

for each $u \in E_{2}$.

By the growth condition $\left(h_{1}\right)$ there is a positive constants $C_{1}$ such that

$$
|F(x, \xi)| \leq C_{1}\left(1+|t|^{q}\right),
$$

for every $x \in \bar{\Omega}, \xi \in \mathbb{R}$. 
Then, by (3.10), (3.13) and using the Sobolev's embedding $X_{0} \hookrightarrow L^{2^{*}}(\Omega)$ we have

$$
\begin{aligned}
J_{K}(u) & \geq \frac{\|u\|_{X_{0}}^{2}}{2}-C_{1}\|u\|_{L^{q}(\Omega)}^{q}-C_{1} \operatorname{meas}(\Omega) \\
& \geq \frac{\|u\|_{X_{0}}^{2}}{2}-C_{1}\|u\|_{L^{2}(\Omega)}^{\beta}\|u\|_{L^{2^{*}(\Omega)}}^{q-\beta}-C_{1} \operatorname{meas}(\Omega) \\
& \geq \frac{\|u\|_{X_{0}}^{2}}{2}-C_{1} \frac{c_{2^{*}}^{q-\beta}}{\lambda_{k_{0}}^{\beta / 2}}\|u\|_{X_{0}}^{q}-C_{1} \operatorname{meas}(\Omega) \\
& \geq\left(\frac{1}{2}-C_{1} \frac{c_{2^{*}}^{q-\beta}}{\lambda_{k_{0}}^{\beta / 2}}\|u\|_{X_{0}}^{q-2}\right)\|u\|_{X_{0}}^{2}-C_{1} \operatorname{meas}(\Omega)
\end{aligned}
$$

for every $u \in E_{2}$.

We may let $\rho:=2 \sqrt{C_{1} \text { meas }(\Omega)+1}$ and choose $k_{0} \in \mathbb{N}$ such that

$$
\lambda_{k_{0}} \geq\left(2^{q} C_{1} c_{2^{*}}^{q-\beta}\left(C_{1} \operatorname{meas}(\Omega)+1\right)^{(q-2) / 2}\right)^{2 / \beta}
$$

to achieve that

$$
J_{K}(u) \geq 1, \quad(\alpha:=1)
$$

for every $u \in E_{2}$ and $\|u\|_{E}=\rho$.

Hence, condition $\left(I_{1}\right)$ is verified. The proof is complete.

Proposition 3.5. The functional $J_{K}$ satisfies condition $\left(I_{2}\right)$.

Proof. Let $W \subset X_{0}$ be a finite-dimensional space. We prove the set

$$
\left\{u \in W: J_{K}(u) \geq 0\right\}
$$

is bounded in $X_{0}$.

Indeed, let $u \in X_{0}$ arbitrary and denote

$$
\Omega_{<}:=\{x \in \Omega:|u(x)|<r\},
$$

as well as

$$
\Omega_{\geq}:=\{x \in \Omega:|u(x)| \geq r\} .
$$

We shall prove that $J_{K}$ satisfies the following estimate:

$$
J_{K}(u) \leq \frac{\|u\|_{X_{0}}^{2}}{2}-\int_{\Omega} \gamma(x)|u(x)|^{\theta} d x+\kappa,
$$

where $\kappa$ is a suitable positive constant and $\gamma \in L^{\infty}(\Omega)$, with $\gamma>0$ in $\Omega$.

To show the above inequality, let us start observing that by $\left(h_{1}\right)$, as pointed out before, the function $F$ satisfies

$$
|F(x, \xi)| \leq C_{1}\left(1+|\xi|^{q}\right), \quad \forall x \in \bar{\Omega}, \forall \xi \in \mathbb{R} .
$$

We claim that there exists $\gamma \in L^{\infty}(\Omega), \gamma>0$ in $\Omega$, such that

$$
F(x, \xi) \geq \gamma(x)|\xi|^{\theta}, \quad \forall x \in \bar{\Omega}, \forall|\xi| \geq r .
$$


Indeed, since $F$ is $\theta$-superhomogeneous, we have that

$$
F(x, \xi) \geq \gamma_{1}(x)|\xi|^{\theta}, \quad \forall x \in \bar{\Omega}, \forall \xi \geq r
$$

where $\gamma_{1}(x):=F(x, r) / r^{\theta}$. It is easy to see that $\gamma_{1} \in L^{\infty}(\Omega)$ and $\gamma_{1}>0$ in $\Omega$.

In a similar way, it follows that

$$
F(x, \xi) \geq \gamma_{2}(x)|\xi|^{\theta}, \quad \forall x \in \bar{\Omega}, \forall \xi \leq-r,
$$

with $\gamma_{2}(x):=F(x,-r) / r^{\theta}$. Also in this case $\gamma_{2} \in L^{\infty}(\Omega)$ and $\gamma_{2}>0$ in $\Omega$.

Then (3.16) holds with

$$
\gamma(x):=\min \left\{\gamma_{1}(x), \gamma_{2}(x)\right\}
$$

for every $x \in \bar{\Omega}$.

Now, by condition (3.15) we conclude that

$$
\int_{\Omega_{<}} F(x, u(x)) d x \geq-C_{1}\left(r^{q}+1\right) \text { meas }(\Omega) .
$$

Further, inequality (3.16) yields

$$
\int_{\Omega_{\geq}} F(x, u(x)) d x \geq \int_{\Omega_{\geq}} \gamma(x)|u(x)|^{\theta} d x .
$$

Then

$$
\begin{aligned}
J_{K}(u) & \leq \frac{\|u\|_{X_{0}}^{2}}{2}-\left(\int_{\Omega_{<}} F(x, u(x)) d x+\int_{\Omega_{\geq}} F(x, u(x)) d x\right) \\
& \leq \frac{\|u\|_{X_{0}}^{2}}{2}-\int_{\Omega_{\geq}} \gamma(x)|u(x)|^{\theta} d x+C_{1}\left(r^{q}+1\right) \text { meas }(\Omega) \\
& \leq \frac{\|u\|_{X_{0}}^{2}}{2}-\int_{\Omega} \gamma(x)|u(x)|^{\theta} d x+\kappa,
\end{aligned}
$$

where

$$
\kappa:=\left(\|\gamma\|_{\infty} r^{\theta}+C_{1}\left(r^{q}+1\right)\right) \operatorname{meas}(\Omega) .
$$

Hence, inequality (3.14) is proved.

At this point, observe that the functional $\|\cdot\|_{\gamma}: X_{0} \rightarrow \mathbb{R}$ defined by

$$
\|u\|_{\gamma}:=\left(\int_{\Omega} \gamma(x)|u(x)|^{\theta} d x\right)^{1 / \theta}
$$

is a norm in $X_{0}$.

Since in $W$ the norms $\|\cdot\|_{X_{0}}$ and $\|\cdot\|_{\gamma}$ are equivalent ( $W$ is finite-dimensional), there exists a positive constant $\kappa_{W}$ such that

$$
\|u\|_{X_{0}} \leq \kappa_{W}\|u\|_{\gamma}
$$

for every $u \in X_{0}$.

Consequently, we have that

$$
J_{K}(u) \leq \frac{\kappa_{W}^{2}}{2}\|u\|_{\gamma}^{2}-\|u\|_{\gamma}^{\theta}+\kappa
$$

for every $u \in W$. 
Hence

$$
\frac{\kappa_{W}^{2}}{2}\|u\|_{\gamma}^{2}-\|u\|_{\gamma}^{\theta}+\kappa \geq 0
$$

for every

$$
u \in\left\{u \in W: J_{K}(u) \geq 0\right\} .
$$

Since $\theta>2$ we conclude that the above set is bounded in $X_{0}$.

Proof of Theorem 3.1 concluded. With the above notations, we can write

$$
X_{0}=E_{1} \oplus E_{2}
$$

where $E_{1}$, given by $\operatorname{Span}\left\{e_{j}: j<k_{0}\right\}$, is the orthogonal complement of $E_{2}$. Thanks to Lemma 3.3 and Propositions 3.4 and 3.5, Theorem 2.1 implies that the functional $J_{K}$ possesses an unbounded sequence of critical value $\left\{J_{K}\left(u_{k}\right)\right\}_{k \in \mathbb{N}}$, where $u_{k}$ is a weak solution of $\left(P_{K}^{f}\right)$. Since $J_{K}^{\prime}\left(u_{k}\right)\left(u_{k}\right)=0$,

$$
\int_{Q}\left|u_{k}(x)-u_{k}(y)\right|^{2} K(x-y) d x d y=\int_{\Omega} f\left(x, u_{k}(x)\right) d x,
$$

and it follows that

$$
J_{K}\left(u_{k}\right)=\int_{\Omega}\left[\frac{1}{2} f\left(x, u_{k}(x)\right) u_{k}(x)-F\left(x, u_{k}(x)\right)\right] d x \rightarrow+\infty,
$$

as $k \rightarrow \infty$. Hence by (3.17) and (3.18) and $\left(h_{2}\right)$, the sequence $\left\{u_{k}\right\}_{k \in \mathbb{N}}$ must be unbounded in $X_{0}$ and in $L^{\infty}(\Omega)$. The proof is complete.

In conclusion, we present a simple and direct application of Theorem 3.1.

Example 3.6. Let $s \in(0,1), n>2 s$ and $\Omega$ be an open bounded set of $\mathbb{R}^{n}$ with Lipschitz boundary and consider the following nonlocal problem:

$$
\begin{cases}-\mathcal{L}_{K} u=u^{3}+u & \text { in } \Omega, \\ u=0 & \text { in } \mathbb{R}^{n} \backslash \Omega,\end{cases}
$$

where $K: \mathbb{R}^{n} \backslash\{0\} \rightarrow(0,+\infty)$ is a map satisfying $\left(k_{1}\right)-\left(k_{3}\right)$.

Owing to Theorem 3.1 , problem $\left(P_{K}\right)$ admits infinitely many weak solutions.

For completeness we also cite the recent papers $[5,7-9,16]$ for some results on elliptic fractional problems.

\section{Acknowledgments}

The paper is realized with the auspices of the GNAMPA Project 2013 entitled: "Problemi non-locali di tipo Laplaciano frazionario". A part of this paper was done while the author was visiting the Department of Mathematics "Guido Castelnuovo" at the University "La Sapienza" in March 2013. He expresses his gratitude to Professor Filomena Pacella for the kind invitation and for many stimulating conversations on the subject of the paper. 


\section{References}

[1] R. Adams, Sobolev Spaces, Academic Press, New York, 1975.

[2] A. Ambrosetti and P. Rabinowitz, Dual variational methods in critical point theory and applications, J. Funct. Anal. 14 (1973), 349-381.

[3] H. Brézis, Analyse fonctionelle, Théorie et applications, Masson, Paris, 1983.

[4] E. Di Nezza, G. Palatucci and E. Valdinoci, Hitchhiker's guide to the fractional Sobolev spaces, Bull. Sci. Math. 136(5) (2012), 521-573.

[5] A. Fiscella, R. Servadei and E. Valdinoci, A resonance problem for non-local elliptic operators, Z. Anal. Anwendungen, 32 (2013), 411-431.

[6] A. Kristály, V. Rădulescu and Cs. Varga, Variational principles in mathematical physics, geometry, and economics: qualitative analysis of nonlinear equations and unilateral problems, Encyclopedia of Mathematics and its Applications, No. 136, Cambridge University Press, Cambridge, 2010.

[7] G. Molica Bisci, Fractional equations with bounded primitive, Appl. Math. Lett. 27 (2014), $53-58$.

[8] G. Molica Bisci and B.A. Pansera, Three weak solutions for nonlocal fractional equations, Adv. Nonlinear Stud. 14 (2014), 591-601.

[9] G. Molica Bisci and R. Servadei, A bifurcation result for nonlocal fractional equations, Anal. Appl. (DOI: 10.1142/S0219530514500067)

[10] P. Pucci and V. Rădulescu, The impact of the mountain pass theory in nonlinear analysis: a mathematical survey, Boll. Unione Mat. Ital. Series IX, 3, (2010), 543-584.

[11] P.H. Rabinowitz, Minimax methods in critical point theory with applications to differential equations, CBMS Reg. Conf. Ser. Math., 65, American Mathematical Society, Providence, RI, 1986.

[12] R. Servadei, The Yamabe equation in a non-local setting, Adv. Nonlinear Anal. 2 (2013), $235-270$.

[13] R. Servadei and E. Valdinoci, Lewy-Stampacchia type estimates for variational inequalities driven by nonlocal operators, Rev. Mat. Iberoam. 29(3) (2013), 1091-1126.

[14] R. Servadei and E. Valdinoci, Mountain Pass solutions for non-local elliptic operators, J. Math. Anal. Appl. 389 (2012), 887-898.

[15] R. Servadei and E. Valdinoci, Variational methods for non-local operators of elliptic type, Discrete Contin. Dyn. Syst. 33(5) (2013), 2105-2137.

[16] R. Servadei and E. Valdinoci, Weak and viscosity solutions of the fractional Laplace equation, Publ. Mat. 58(1) (2014), 133-154.

[17] R. Servadei and E. Valdinoci, The Brézis-Nirenberg result for the fractional Laplacian, to appear in Trans. AMS.

[18] M. Struwe, Variational methods, Applications to nonlinear partial differential equations and Hamiltonian systems, Ergebnisse der Mathematik und ihrer Grenzgebiete, 3, Springer-Verlag, Berlin-Heidelberg, 1990.

Dipartimento P.A.U., Università degli Studi "Mediterranea" di Reggio Calabria, Salita

Melissari - FeO di Vito, 89100 Reggio CAlabria, Italy

E-mail address: gmolica@unirc.it 
